\title{
Efficacy of Computed Tomography-Based Navigation for Cup Placement in Revision Total Hip Arthroplasty
}

\author{
Yuta Kubota, MD, Nobuhiro Kaku, MD, Tomonori Tabata, MD, \\ Hiroaki Tagomori, MD, Hiroshi Tsumura, MD \\ Department of Orthopaedic Surgery, Faculty of Medicine, Oita University, Oita, Japan
}

\begin{abstract}
Background: Navigation systems are an effective tool to improve the installation accuracy of the cup in primary total hip arthroplasty. This study aimed to evaluate the efficacy of a computed tomography-based navigation system in achieving optimal installation accuracy of implants in revision total hip arthroplasty and to clarify the usefulness of the navigation system.

Methods: We conducted a retrospective study of 23 hips in 23 patients who underwent revision total hip arthroplasty using a computed tomography-based navigation system; the control group comprised 33 hips in 33 patients who underwent revision total hip arthroplasty without a navigation system.

Results: The average cup position with the navigation system was $40.0^{\circ} \pm 3.7^{\circ}$ in radiographic abduction angle, $18.8^{\circ} \pm 4.8^{\circ}$ in radiographic anteversion, and $41.2^{\circ} \pm 8.9^{\circ}$ in combined anteversion; without the navigation system, the average cup position was $38.7^{\circ} \pm 6.1^{\circ}, 19.0^{\circ} \pm 9.1^{\circ}$, and $33.6^{\circ} \pm 20.5^{\circ}$, respectively. The achievement rate of cup positioning within the Lewinnek safe zone was not significantly different between the navigation group (82.6\%) and control group (63.6\%). In contrast, the achievement rate of cup positioning within the Widmer combined anteversion guidelines was significantly greater in the navigation group (78.3\%) than in the control group $(48.0 \%, p=0.029)$. Furthermore, outlier cases in the navigation group had a smaller variance of deviation from the optimal cup position than those in the control group did.

Conclusions: The results show that the use of navigation for revision total hip arthroplasty improved cup positioning and reduced the range of outliers. Improvement of cup placement accuracy influenced the installation of the stem and also improved the achievement rate of combined anteversion. Thus, a computed tomography-based navigation system is very useful for surgeons when placing the cup within the target angle in revision total hip arthroplasty.

Keywords: Hip, Arthroplasty, Computers, Tomography, Revision
\end{abstract}

The main reason for revision total hip arthroplasty (THA) in the United States is dislocation. ${ }^{1)}$ Optimal cup and stem positions are important to prevent impingement and dislocation after surgery. Although it is difficult to demonstrate

Received May 17, 2018; Accepted November 12, 2018

Correspondence to: Nobuhiro Kaku, MD

Department of Orthopaedic Surgery, Faculty of Medicine, Oita University,

1-1 Idaigaoka, Hazama-machi, Oita 879-5593, Japan

Tel: +81-975865872, Fax: +81-975866647

E-mail: nobuhiro@oita-u.ac.jp a significant effect of implant cup position, as many factors in clinical practice influence outcomes, some clinical reports have stated that the cup position affects polyethylene wear, as well as the survival rate. ${ }^{2)}$

In revision surgery, preoperative planning is challenging, as it is difficult to visualize the detailed bone surface because of metal artifacts. Furthermore, determining the anatomical orientation is problematic because of bone loss. In addition, it is often technically difficult to perform revision surgery; thus, the dislocation rate after revision surgery is even higher than that after primary surgery. ${ }^{3)}$ 
Therefore, an appropriate and accurate implant position is critical in revision surgery. Efforts have been made to improve implant position accuracy with detailed planning using preoperative two-dimensional (2D) or three-dimensional (3D) templates, intraoperative alignment guides, and X-ray radiography., ${ }^{4,5)}$ In recent years, navigation has emerged as a method to improve implant position accuracy, and the precision of navigation has been noted in primary surgery reports. ${ }^{6,7)}$ Computed tomography (CT)based navigation performs accurate and precise registration $;^{8)}$ however, there are concerns that metal artifacts in $\mathrm{CT}$ imaging affect implant position and registration. It is of interest to achieve the same accuracy in revision surgery as that in primary surgery using CT-based navigation. However, until now, there have been no studies comparing revision THA with and without navigation at the same institution and evaluating the postoperative combined anteversion of the cup and femoral stem using navigation system for revision THA. The purpose of this study was to evaluate the installation accuracy of implants with and without a CT-based navigation system in revision THA and to clarify the usefulness of the CT-based navigation system.

\section{METHODS}

This retrospective study was approved by the Institutional Review Board at Oita University, and all study participants provided informed consent at the follow-up examination. From February 2010 to October 2016, we performed a total of 150 revision THA operations. Of these cases, we targeted 56 revision cases that were both acetabular and femoral component revisions, but we excluded 94 isolated acetabular revision and isolated stem revision cases. These operations were performed through a posterolateral approach, and a cup was inserted before stem insertion.

We retrospectively reviewed 23 hips in 23 patients (18 females and 5 males) who underwent revision THA using a CT-based navigation system (VectorVision compact hip CT version 3.5.2; Brainlab, Munich, Germany) between February 2010 and October 2016; the control group consisted of 33 hips in 33 patients ( 28 females and 5 males) who underwent revision THA through a posterolateral approach on lateral position without navigation before February 2010. As the navigation procedure is cumbersome for the femoral bone side in the lateral position, we have used only CT-based navigation for the acetabular side in revision THA surgery since February 2010. In both groups, we removed cup and stem implants first. Then, we inserted the cup before insertion of the femoral stem. The indications for revision THA included cup loosening in 43 cases, infection in eight cases, recurrent dislocation in three cases, unknown pain in one case, and periprosthetic fracture in one case. CT-based navigation for revision THA surgery has been used at our institution since February 2010. Patient demographic data are provided in Table 1 . With the exception of operative time and observation period, there were no significant group differences in the evaluated background parameters. The utilized stems and cups are listed in Table 2.

\section{Preoperative Planning}

For preoperative planning, CT images from the iliac wing to the knee joint were acquired using a helical CT scanner (Aquilion CX; Toshiba Medical Systems Corporation, Tokyo, Japan) with 1-mm slice thickness. CT data were transferred to the planning module, which was then used to determine the optimal component size, angle, and position. Our goal was to implant the acetabular component with a radiographic abduction angle of $40^{\circ}$, radiographic anteversion of $15^{\circ}$, and radiographic combined anteversion of the cup and femoral stem of $37^{\circ}$ using Widmer's equation [cup anteversion $+(0.7 \times$ stem anteversion $)] .^{9)}$

\section{Intraoperative Procedures: Navigation Group}

All surgeries were performed by one surgeon (NK) using a posterolateral approach. The VectorVision CT-based navigation system was used to determine cup and stem alignments. We performed surface registration after the induction of general anesthesia. We inserted two screws and placed the antenna on the iliac crest while the patient was in the lateral decubitus position; then, two fluoroscopic pelvic images, recorded from different angles of $>20^{\circ}$, were obtained using a mobile fluoroscopy system (Philips

\begin{tabular}{|lcc|}
\hline \multicolumn{1}{|c|}{ Tahle 1. Patient Demographic Data } \\
\hline \multicolumn{1}{|c|}{ Variable } & $\begin{array}{c}\text { Control group } \\
(\mathrm{n}=33)\end{array}$ & $\begin{array}{c}\text { Navigation group } \\
(\mathrm{n}=23)\end{array}$ \\
\hline Age $(\mathrm{yr})$ & $65.6 \pm 13.6$ & $71.7 \pm 6.92$ \\
\hline Sex (male:female) & $5: 28$ & $5: 18$ \\
\hline Body mass index $\left(\mathrm{kg} / \mathrm{m}^{2}\right)$ & $22.7 \pm 3.85$ & $24.4 \pm 3.95$ \\
\hline Bleeding (mL) & $789.53 \pm 641.32$ & $862.17 \pm 659.78$ \\
\hline Operative time $(\mathrm{min})$ & $257.13 \pm 82.45$ & $345.65 \pm 124.20$ \\
\hline Observation period $(\mathrm{mo})$ & $80.6 \pm 38.0$ & $42.2 \pm 23.2$ \\
\hline Head size $(\mathrm{mm})$ & 29.3 & 31.1 \\
\hline
\end{tabular}

Values are presented as mean \pm standard deviation. 
Table 2. Product Name and Case Number

\begin{tabular}{|c|c|}
\hline Stem ( 56 cases) & Cup (56 cases) \\
\hline Stryker Exeter total hip system (Stryker, Kalamazoo, MI, USA): 25 cases & K-MAX KT plate S + Crossfire polyethylene (Stryker): 20 cases \\
\hline S-ROM total hip system (DePuy, Warsaw, IN, USA): 20 cases & K-MAX KT plate S + standard socket CP (JMM, Kyoto, Japan): 14 cases \\
\hline SC hip system (Kyocera, Kyoto, Japan): 4 cases & Pinnacle (DePuy): 7 cases \\
\hline VerSys cemented revision/calcar hip system (Zimmer, Warsaw, IN, USA): 4 cases & RingLoc (Biomet): 6 cases \\
\hline Arcos modular femoral revision system (Biomet, Warsaw, IN, USA): 2 cases & TriAD HA PSL (Stryker): 5 cases \\
\hline \multirow[t]{2}{*}{ Bi-Metric hip system (Biomet): 1 case } & AMS HA Shell (JMM): 2 cases \\
\hline & Continuum Acetabular System (Zimmer): 2 cases \\
\hline
\end{tabular}

BV-29 C-Arm; Koninklijke Philips N.V., Eindhoven, the Netherlands), with the patient in the same position. The fluoroscopic anterior-posterior image included the pubic symphysis, superior and inferior pubic ramus, and ischium around the obturator foramen. Surface matching was performed by touching two points: the superior iliac spine and iliac crest. The position of the pubic symphysis was finely adjusted and calibrated on the computer monitor, and the accuracy of calibration was confirmed within 2 $\mathrm{mm}$ preoperatively. After calibrating the cup, it was placed during the surgery.

\section{Intraoperative Procedures: Control Group}

We measured the installed cup and stem angles on CT before surgery. The patient's pelvis was set exactly vertical on the operating table. During the operation, just before the implants were removed, we marked their angle line on the pelvis and femur, using a surgical sheet. Considering the marks as landmarks, we set the new implant; cup placement was determined using the common ailment guide attached to the cup and landmarks together.

\section{Postoperative Results}

Postoperative cup angles were measured on postoperative $\mathrm{X}$-rays using the planning module (2D template; Kyocera, Kyoto, Japan). Femoral stem anteversion was evaluated using CT. Femoral stem anteversion was defined as the angle formed between the proximal femoral stem axis and a line tangential to the bilateral posterior femoral condylar margin on the axial plane. The achievement rate for target angle was researched in radiographic abduction and anteversion of the cup and Widmer combined anteversion, respectively. We used 22 cementless cups, 34 cement cups, 23 cementless stems, and 33 cement stems.

\section{Statistical Analysis}

This report is a retrospective research that was not randomized and evaluated the surgical duration, amount of bleeding, cup and stem angles, combined anteversion, postoperative dislocation, and re-revision rate. For the cup position, a 2D template was used for both cement and cementless cups. Statistical analyses were performed using IBM SPSS ver. 22.0 (IBM Corp., Armonk, NY, USA). Independent-samples $t$-tests were used to compare the surgical duration, amount of bleeding, and accuracy of cup positioning between groups. One-way analysis of variance was also performed using the Tukey test for intergroup comparisons. We used the chi-square test to compare the planned concordance rate between groups. A $p$-value < 0.05 indicated statistical significance in all analyses.

\section{RESULTS}

In the total patient cohort, the average surgical duration was $294 \pm 110$ minutes (range, 90 to 622 minutes), the average intraoperative bleeding volume was $820 \pm 644 \mathrm{~mL}$ (range, 180 to $3,200 \mathrm{~mL}$ ), and the average ball head size was $\varphi 30.0 \mathrm{~mm}(\varphi 22 \mathrm{~mm}, \mathrm{n}=1 ; \varphi 26 \mathrm{~mm}, \mathrm{n}=4 ; \varphi 28 \mathrm{~mm}$, $\mathrm{n}=24 ; \varphi 30 \mathrm{~mm}, \mathrm{n}=2 ; \varphi 32 \mathrm{~mm}, \mathrm{n}=19$; and $\varphi 36 \mathrm{~mm}, \mathrm{n}$ $=6$ ). There were some intraoperative complications that occurred in one joint with a greater trochanter fracture. Postoperative complications included delayed wound healing in one case and deep infection in one case. Postoperative dislocation occurred in one case in the navigation group and in one case in the control group (Table 3). The cup was positioned within the safe zone in the dislocation case of the navigation group. In the dislocation case of the control group, the cup was positioned outside of both the Lewinnek safe zone and the combined anteversion safe zone. The dislocations did not reoccur, and there was no re-revision surgery. 
The rates of the radiographic abduction angle alone and both the radiographic abduction and anteversion were within the Lewinnek safe zone ${ }^{10)}$ were not significantly greater in the navigation group than in the control group ( $p$ $=0.137$ and $p=0.122$, respectively) (Table 4 ). In the navigation group (radiographic abduction angle, $40^{\circ} \pm 10^{\circ}$; radiographic anteversion, $15^{\circ} \pm 10^{\circ}$ ), the radiographic abduction angle was within the Lewinnek safe zone in $100 \%$ of cases (23/23), whereas the radiographic anteversion was within the Lewinnek safe zone in $82.6 \%$ of cases (19/23) (Fig. 1). Overall, in the control group, the radiographic abduction angle was within the Lewinnek safe zone in $90.9 \%$ of cases (30/33), whereas the radiographic anteversion was within the Lewinnek safe zone in $69.7 \%$ of cases $(23 / 33)$ (Fig. 2). Overall, $82.6 \%$ of cups (19/23) were placed in the safe zone for both the radiographic abduction angle and radiographic anteversion in the navigation group, and $63.6 \%$ of cups (21/33) were placed in the safe zone for both

\section{Table 3. Dislocation Cases}

\begin{tabular}{lcc}
\hline \multicolumn{1}{c}{ Variable } & Case 1 & Case 2 \\
\hline Navigation & No & Use \\
\hline Duration $(\mathrm{mo})$ & 0.5 & 38 \\
\hline Head size $(\mathrm{mm})$ & 28 & 32 \\
\hline Radiographic abduction angle $\left(^{\circ}\right)$ & 41.80 & 42.30 \\
\hline Radiographic anteversion $\left({ }^{\circ}\right)$ & 4.80 & 21.70 \\
\hline Widmer combined anteversion $\left({ }^{\circ}\right)$ & 19.78 & 43.90 \\
\hline
\end{tabular}

the radiographic abduction angle and radiographic anteversion in the control group, without a significant group difference $(p=0.122)$.

In contrast, in terms of the combined anteversion, the target, defined as $37^{\circ} \pm 10^{\circ}$ (see Preoperative Planning), was achieved more often in the navigation group than in the control group $(p=0.029)$. The cup position was considered optimal in $78.3 \%$ of cases $(18 / 23)$ in the navigation group and in $48.0 \%$ of cases (13/27) in the control group $(p=0.029)$.

Deviations from the median value of the cup position for the cases that did not fall within the cup position range are summarized in Table 5. The navigation group had a smaller variance of deviation from the optimal cup

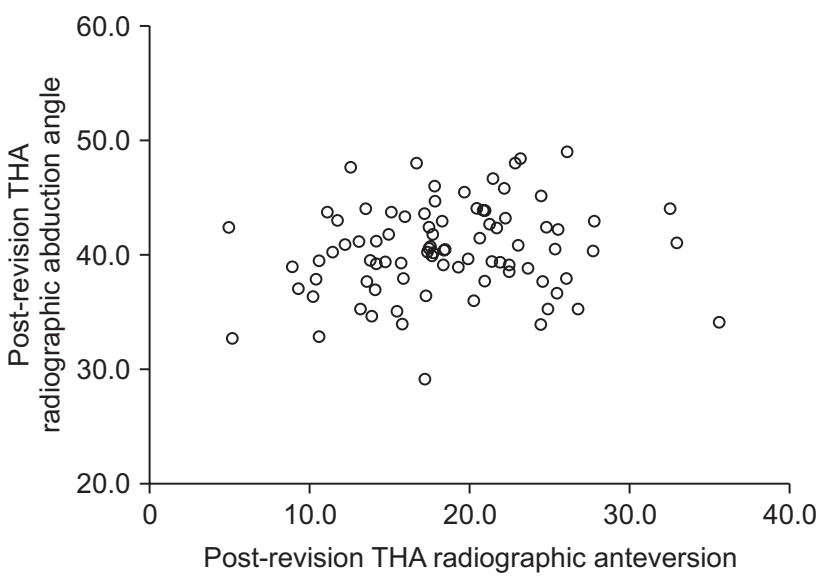

Fig. 1. Safe zone outliers after revision total hip arthroplasty (THA) with navigation.

Table 4. Both Component (Acetabular Component and Femoral Component) Revision

\begin{tabular}{|c|c|c|c|}
\hline Variable & Control group $(n=33)$ & Navigation group $(n=23)$ & $\begin{array}{l}\text { Safe zone } p \text {-value } \\
\text { (average } p \text {-value) }\end{array}$ \\
\hline Radiographic abduction angle (\%) & $90.9(30 / 33)$ & $100(23 / 23)$ & $0.137(0.364)$ \\
\hline Mean $\pm \operatorname{SD}\left({ }^{\circ}\right)$ & $38.66 \pm 6.11$ & $39.97 \pm 3.69$ & \\
\hline Radiographic anteversion (\%) & $69.7(23 / 33)$ & $82.6(19 / 23)$ & $0.122(0.901)$ \\
\hline Mean $\pm \operatorname{SD}\left({ }^{\circ}\right)$ & $19.03 \pm 9.05$ & $18.77 \pm 4.83$ & \\
\hline Both radiographic abduction angle and radiographic anteversion (\%) & $63.6(21 / 33)$ & $82.6(19 / 23)$ & 0.122 \\
\hline Widmer combined anteversion (\%) & $48.0(13 / 27)$ & $78.3(18 / 23)$ & $0.029(0.098)$ \\
\hline Mean $\pm \operatorname{SD}\left({ }^{\circ}\right)$ & $33.55 \pm 20.53$ & $41.23 \pm 8.94$ & \\
\hline Stem anteversion & & & 0.151 \\
\hline Mean $\pm \operatorname{SD}\left({ }^{\circ}\right)$ & $14.82 \pm 11.99$ & $19.23 \pm 9.81$ & \\
\hline
\end{tabular}

Values are presented as percent (number of cases).

SD: standard deviation. 
Kubota et al. Computed Tomography-Based Navigation in Revision Total Hip Arthroplasty

Clinics in Orthopedic Surgery • Vol. 11, No. 1, 2019•www.ecios.org

position than the control group (Fig. 3).

\section{DISCUSSION}

In revision THA, detailed preoperative planning and determination of the anatomical orientation are difficult because of bone loss during surgery. Reconstruction, such as soft tissue tension adjustment, rather than primary surgery is often technically difficult. An optimal implant cup position can prevent many complications, such as dislocation, implant failure, and wear, which affect the overall clinical results. ${ }^{11)}$ The present study demonstrates the usefulness of a CT-based navigation system in improving the cup position accuracy in revision THA.

Regarding the optimal cup position, Lewinnek et al. $^{10)}$ defined the safe zone in terms of the cup alignment as $40^{\circ} \pm 10^{\circ}$ in the abduction angle and $15^{\circ} \pm 10^{\circ}$ in the anteversion angle. Widmer and Zurfluh ${ }^{9)}$ reported that a combined anteversion comprising the radiographic anteversion of the cup and stem torsional angle can be derived using the finite element method. They concluded that the sum

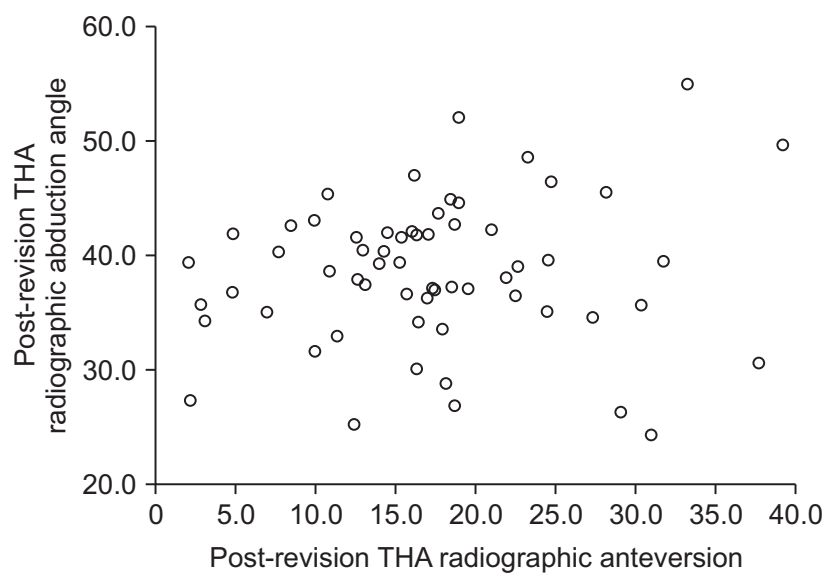

Fig. 2. Safe zone outliers after revision total hip arthroplasty (THA) without navigation. of the cup anteversion and 0.7 times the stem anteversion should equal $37^{\circ}$. Yoshimine ${ }^{12)}$ defined the safe zones for the combined cup ( $\beta$ anat: anatomic anteversion) and neck anteversion (b) as the areas that fulfill all range of motion criteria, without prosthetic impingement. They concluded that the optimal values of the combined cup and neck anteversions can be estimated using the following formula: $\alpha$ (radiographic abduction) $+\beta$ anat $+0.77(b)=84.3$. Recent studies by Sadhu et al. ${ }^{13)}$ have shown that the use of the Lewinnek safe zone is effective for preventing dislocation in both primary and revision cases. Furthermore, Dorr et al. ${ }^{14)}$ showed that the achievement rate using a navigation system for primary THA was $96 \%$ (45/47 cases), with an average value of $37.6^{\circ} \pm 7^{\circ}$ (range, $19^{\circ}$ to $50^{\circ}$ ), when the safe zone of the combined anteversion was $25^{\circ}-50^{\circ}$. In the present study, we decided to use the Lewinnek safe zone and Widmer combined anteversion guidelines to verify the accuracy of cup placement when using navigation.

Minoda et al. ${ }^{15)}$ reported that when the cup was manually placed in its target angle for primary THA, defined as $45^{\circ}$ in radiographic abduction angle and $20^{\circ}$ in radiographic anteversion, the possibility that the radiographic abduction angle and radiographic anteversion are

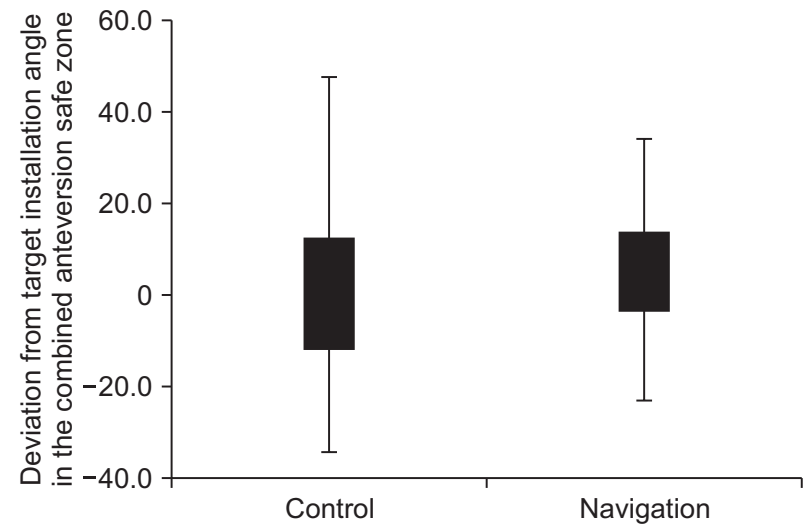

Fig. 3. Deviation from the optimal cup position.

Table 5. Outlier Cases

\begin{tabular}{|c|c|c|}
\hline Variable & Navigation group $(n=23)$ & Control group $(n=33)$ \\
\hline Radiographic abduction angle & 0 & 3 \\
\hline Mean \pm SD (range), ${ }^{\circ}$ & - & $13.80 \pm 0.82(14.70-13.10)$ \\
\hline Radiographic anteversion & 4 & 10 \\
\hline Mean \pm SD (range) ${ }^{\circ}$ & $11.98 \pm 1.09(10.50-12.80)$ & $15.35 \pm 5.37(10.20-24.30)$ \\
\hline Widmer combined anteversion & 5 & 14 \\
\hline Mean \pm SD (range), ${ }^{\circ}$ & $15.21 \pm 6.47(11.62-26.69)$ & $23.4 \pm 11.3(0.76-39.53)$ \\
\hline
\end{tabular}


within the optimal cup position of $\pm 10^{\circ}$ was $86.7 \%$ and $82.9 \%$, respectively; the combined anteversion achievement rate was $72.2 \%$ and the angular deviation was $5.3^{\circ}$ for both. According to Bosker et al., ${ }^{16)}$ the achievement rates for cup placement in the Lewinnek safe zone for primary THA were $85.2 \%$ and $82.7 \%$ in radiographic abduction and radiographic anteversion, respectively. In this previous report, the achievement rate for a target angle within $\pm 5^{\circ}$ was as low as $21.5 \%$, which the authors reported as a limitation in manual cup positioning. Manual accuracy in revision surgery is achieved when the optimal cup position is set to $30^{\circ}-45^{\circ}$ in the radiographic abduction angle and $5^{\circ}-25^{\circ}$ in the radiographic anteversion. Callanan et al. ${ }^{11)}$ reported on 170 hip revision surgeries and found that 120 cases $(70.6 \%)$ were within the safe zone in terms of radiographic abduction, $128(75.3 \%)$ were within the safe zone in terms of radiographic anteversion, and 97 (57.1\%) were within the safe zones in terms of both radiographic abduction and radiographic anteversion. Manual placement in revision THA is even lower than that in primary THA. Thus, tools are needed to improve accuracy in revision THA.

The navigation system is a support tool for improving cup position accuracy. Numerous studies have shown the utility of navigation systems in primary THA. Kalteis et al. ${ }^{7)}$ reported that $14(46.7 \%)$ of 30 cases of primary THA performed freehand achieved placement of the cup within the safe zone (deviation angle: radiographic abduction angle, $6.1^{\circ}$; radiographic anteversion, $13.0^{\circ}$ ). However, among the 30 cases using CT-based navigation, 25 (83.3\%) were within the safe zone (deviation angle: radiographic abduction angle, $4.2^{\circ}$; radiographic anteversion, $5.3^{\circ}$ ). Sugano et al. ${ }^{17)}$ evaluated 120 hips of freehand THA, 60 hips of THA with navigation: all cups with navigation were placed within the Lewinnek safe zone; 31 cups $(25.8 \%)$ without navigation were placed outside this zone; and the dislocation rate after surgery was seven in all freehand cases and zero in CT-based navigation cases.

Reported cases of cup placement accuracy and dislocation rates in revision THA using navigation are shown in Table 6 . There are few reports on cup placement accuracy and dislocation rate in revision THA using navigation. Because bony surface outlines around the acetabulum are radiographically unclear due to metal artifacts and bone atrophy from prosthesis, it is technically difficult to perform accurate segmentation at the time of preoperative planning and landmark registration using navigation during the surgery. Furthermore, because of the problems that arise with the use of a navigation system in revision THA, it is uncertain whether the acetabular cup position achieves similar accuracy as that obtained in primary THA. However, Nakamura et al. ${ }^{18)}$ and Kuroda et al. ${ }^{19)}$ achieved the same accuracy with CT-based navigation for primary and revision THA. Additionally, in a study by Chang et al.,20) the optimal cup position was achieved with a very high probability: achievements in radiographic abduction and that in combined anteversion were both $100 \%$. Our study is the first to report the cup position accuracy in CT-based navigation for revision THA in comparison to that in a manual control group. Additionally, the present study is the first to report the cup position accuracy in terms of the combined anteversion in CT-based navigation for revision THA compared to that achieved with manual placement. Overall, the precision was high for radiographic abduction and combined anteversion in the navigation group, and the use of a navigation system was sufficiently effective for improving accuracy, if the stem rotation could be controlled intraoperatively with the cup position.

This study targeted only total revision cases and excluded isolated cup or stem revision cases because we would like to evaluate correctly the influence of more precise cup angles with navigation for femoral stem insertion to obtain the appropriate combined anteversion. When the cup anteversion is out of the target angle after its final impaction, the surgeon would try to adjust the stem anteversion to achieve the target combined anteversion. Nevertheless, the implanted stem anteversion was near in both the navigation and control groups. It is easier to implant the femoral stem in the posterolateral approach than in other approaches. However, it is very difficult to adjust the intraoperative stem anteversion because of the femoral shape and stem implant stability. ${ }^{21)}$ Furthermore, Widmer's equation [cup anteversion $+(0.7 \times$ stem anteversion $)$ ] means that if we try to add Widmer combined anteversion, we must place the stem implant angle multiplied by 1.42 mathematically. Moreover, adjusting the stem anteversion to obtain a large difference from the target angle of the cup is difficult.

In this study, the achievement rate shows that cups were placed in the safe zone without a significant difference between the navigation group and the control group. On the contrary, the combined anteversion was more appropriate in the navigation group than in the control group. In the navigation group, it was thought that the range of error of the anteversion angle cup was only small to enable adjustment by controlling on the femoral stem side. In contrast, since the cup anteversion error was large in the control group, we believe that it is difficult to insert within the appropriate combined anteversion by adjust- 
Kubota et al. Computed Tomography-Based Navigation in Revision Total Hip Arthroplasty

Clinics in Orthopedic Surgery • Vol. 11, No. 1, 2019• www.ecios.org

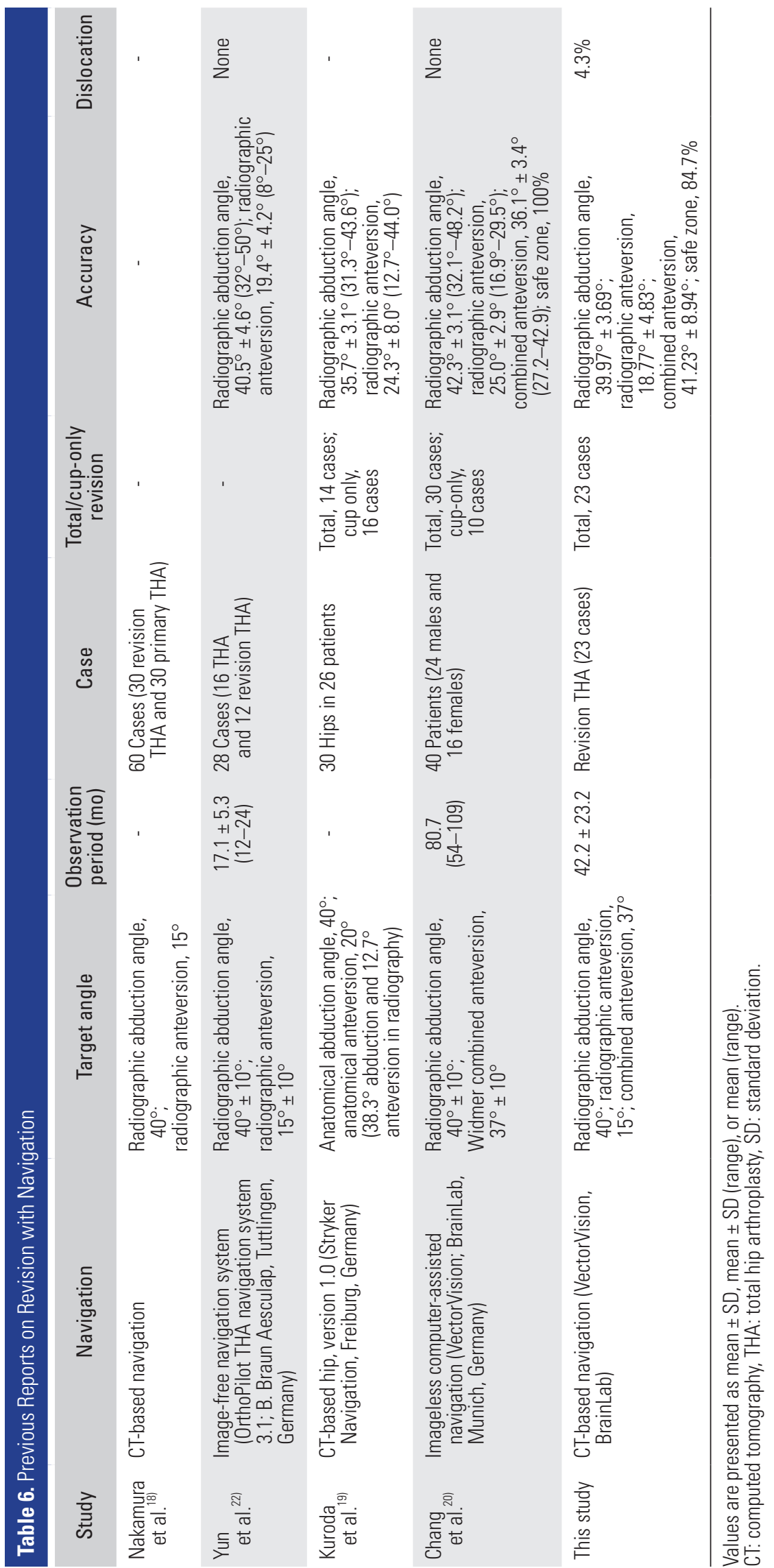


ing the stem angle based on our results. Another reason is that we were able to know the accurate difference digitally from the target angle in real time intraoperatively and adjust the femoral stem more accurately using the definite deviation data as a reference in the navigation group. Contrary to this, since the installation angle of the cup was usually estimated visually and ambiguously in the control group without navigation, adjustment of the stem anteversion also becomes inaccurate than in the navigation group. The use of navigation for cup alone influences the installation of the stem, and there are fewer variations in the femoral stem anteversion in the navigation group than in the control group. Therefore, increasing the accuracy of the cup installation angle using navigation even if without using navigation on the femoral stem side is useful from the viewpoint of combined anteversion.

According to Kuroda et al., ${ }^{19)}$ the postoperative dislocation rate can be $6.67 \%$, which is similar to that in primary THA using CT-based navigation. In our study, the control (3.0\%) and navigation (4.3\%) groups had almost the same postoperative dislocation incidence rates, and an advantage of the use of a navigation system for avoiding postoperative dislocation was not shown. In revision THA, the adjustment of the soft tissue balance (leg length correction/tissue preservation/repair) is more difficult than that in primary THA. Patients who undergo revision THA are older than those who undergo primary THA; therefore, they tend to fall. In the present study, both dislocation cas- es were caused by falls and were not repeated. There was no re-revision surgery in either group. Thus, the results reconfirm that many factors involved in cup position and soft tissue tension affect dislocation in revision THA, and patient education is very important.

The present study has limitations. The present study was not a randomized comparative study performed with or without navigation during the same time period, and additional clinical evaluation is needed. Another limitation is that this study targeted only total revision cases; thus, the number of cases was small. In conclusion, this study is the first to evaluate CA in revision THA with and without navigation. It is meaningful to calculate and compare carefully each setting angle of implants and the achievement rate of target range in both groups. Using navigation, cup placement accuracy was improved, and the range of outliers was reduced. Improvement of cup placement accuracy influenced the installation of the stem and also improved the accuracy of CA. Thus, a CT-based navigation system is very useful for surgeons to achieve high precision in placement of the cup within the target angle during revision THA.

\section{CONFLICT OF INTEREST}

No potential conflict of interest relevant to this article was reported.

\section{REFERENCES}

1. Bozic KJ, Kurtz SM, Lau E, Ong K, Vail TP, Berry DJ. The epidemiology of revision total hip arthroplasty in the United States. J Bone Joint Surg Am. 2009;91(1):128-33.

2. Del Schutte H Jr, Lipman AJ, Bannar SM, Livermore JT, Ilstrup D, Morrey BF. Effects of acetabular abduction on cup wear rates in total hip arthroplasty. J Arthroplasty. 1998;13(6):621-6.

3. Lachiewicz PF, Soileau E, Ellis J. Modular revision for recurrent dislocation of primary or revision total hip arthroplasty. J Arthroplasty. 2004;19(4):424-9.

4. Digioia AM 3rd, Jaramaz B, Plakseychuk AY, et al. Comparison of a mechanical acetabular alignment guide with computer placement of the socket. J Arthroplasty. 2002;17(3):359-64.

5. Gross TP, Liu F, Webb L. Intraoperative radiographs for placing acetabular components in hip resurfacing arthroplasty. Clin Orthop Relat Res. 2011;469(6):1554-9.
6. Kitada M, Nakamura N, Iwana D, Kakimoto A, Nishii T, Sugano N. Evaluation of the accuracy of computed tomography-based navigation for femoral stem orientation and leg length discrepancy. J Arthroplasty. 2011;26(5):674-9.

7. Kalteis T, Handel M, Bathis H, Perlick L, Tingart M, Grifka J. Imageless navigation for insertion of the acetabular component in total hip arthroplasty: is it as accurate as CT-based navigation? J Bone Joint Surg Br. 2006;88(2):163-7.

8. Yamada K, Endo H, Tetsunaga T, Miyake T, Sanki T, Ozaki T. Accuracy of cup positioning with the computed tomography-based two-dimensional to three-dimensional matched navigation system: a prospective, randomized controlled study. J Arthroplasty. 2018;33(1):136-43.

9. Widmer KH, Zurfluh B. Compliant positioning of total hip components for optimal range of motion. J Orthop Res. 2004;22(4):815-21.

10. Lewinnek GE, Lewis JL, Tarr R, Compere CL, Zimmerman JR. Dislocations after total hip-replacement arthroplasties. J 
Kubota et al. Computed Tomography-Based Navigation in Revision Total Hip Arthroplasty

Clinics in Orthopedic Surgery • Vol. 11, No. 1, 2019• www.ecios.org

Bone Joint Surg Am. 1978;60(2):217-20.

11. Callanan MC, Jarrett B, Bragdon CR, et al. The John Charnley Award: risk factors for cup malpositioning: quality improvement through a joint registry at a tertiary hospital. Clin Orthop Relat Res. 2011;469(2):319-29.

12. Yoshimine F. The safe-zones for combined cup and neck anteversions that fulfill the essential range of motion and their optimum combination in total hip replacements. J Biomech. 2006;39(7):1315-23.

13. Sadhu A, Nam D, Coobs BR, Barrack TN, Nunley RM, Barrack RL. Acetabular component position and the risk of dislocation following primary and revision total hip arthroplasty: a matched cohort analysis. J Arthroplasty. 2017;32(3):987-91.

14. Dorr LD, Malik A, Dastane M, Wan Z. Combined anteversion technique for total hip arthroplasty. Clin Orthop Relat Res. 2009;467(1):119-27.

15. Minoda Y, Kadowaki T, Kim M. Acetabular component orientation in 834 total hip arthroplasties using a manual technique. Clin Orthop Relat Res. 2006;445:186-91.

16. Bosker BH, Verheyen CC, Horstmann WG, Tulp NJ. Poor accuracy of freehand cup positioning during total hip ar- throplasty. Arch Orthop Trauma Surg. 2007;127(5):375-9.

17. Sugano N, Takao M, Sakai T, Nishii T, Miki H. Does CT-based navigation improve the long-term survival in ceramic-on-ceramic THA? Clin Orthop Relat Res. 2012;470(11):3054-9.

18. Nakamura N, Nishii T, Kitada M, Iwana D, Sugano N. Application of computed tomography-based navigation for revision total hip arthroplasty. J Arthroplasty. 2013;28(10):1806-10.

19. Kuroda K, Kabata T, Maeda T, et al. The value of computed tomography based navigation in revision total hip arthroplasty. Int Orthop. 2014;38(4):711-6.

20. Chang JD, Kim IS, Prabhakar S, Mansukhani SA, Lee SS, Yoo JH. Revision total hip arthroplasty using imageless navigation with the concept of combined anteversion. J Arthroplasty. 2017;32(5):1576-80.

21. Bargar WL, Jamali AA, Nejad AH. Femoral anteversion in THA and its lack of correlation with native acetabular anteversion. Clin Orthop Relat Res. 2010;468(2):527-32.

22. Yun HH, Yoon JR, Yu JJ, Seo HS. Navigated acetabular cup fixation for acetabular deformity or revision total hip arthroplasty. Hip Pelvis. 2014;26(3):150-6. 